\title{
Damage Localization Test of Civil GFRP Material Based on Acoustic Emission
}

\author{
Yanhua She ${ }^{1 *}$, Gaojie Cai ${ }^{1}$ \\ ${ }^{1}$ School of Urban Construction, Yangtze University, Jingzhou 434023, China
}

\author{
DOI: $\underline{10.36348 / \text { sjet.2021.v06i02.003 }}$ \\ | Received: 09.02.2021 | Accepted: 22.02.2021 | Published: 23.02.2021 \\ *Corresponding author: Yanhua She
}

\section{Abstract}

To detect the damage location, the acoustic emission sensor is fixed on the surface of civil GFRP tube by the external bonding method. And damage position of the GFRP Tube under static loading is studied. The acoustic emission sensor is used to receive the signal of the GFRP tube rupture under static loading, and the data is processed by using the acoustic emission damage location principle to realize the real-time display of the specific location of the damage point on the specimen. It is found that the position shown by the test data is approximately the same as the actual damage position of the GFRP tube. The test indicates that the damage position of GFRP tube under static force can be displayed in real time in three dimensions through acoustic emission technology.

Keywords: GFRP, Acoustic emission, Static loading, Damage location detection.

Copyright $($ ) 2021 The Author(s): This is an open-access article distributed under the terms of the Creative Commons Attribution 4.0 International License (CC BY-NC 4.0) which permits unrestricted use, distribution, and reproduction in any medium for non-commercial use provided the original author and source are credited.

\section{INTRODUCTION}

Glass Fiber Reinforced Plastic (GFRP) is a new functional material produced by the composite process of synthetic resin and glass fiber [1]. It has the advantages of light weight, high strength, heat resistance, good corrosion resistance and insulation, low production energy consumption and environmentally friendly [2]. Therefore, GFRP has gradually become the potential material in structural design of water conservancy, civil engineering and road engineering [3$5]$, especially widely used in various concrete structures [6-9].

At present, many scholars are devoted to the research of acoustic emission calculation. Ji et al., [10] proved the existence of fractal in concrete acoustic emission process through experiments. $\mathrm{Xu}$ et al., [11] used acoustic emission to reveal the damage degree of FRP concrete structure under external loads. Li et al., [12] analyzed the acoustic emission characteristics of tensile damage of FRP composites by three-dimensional parameter method, and revealed the development, evolution process and law of tensile damage of FRP composites at different angles macroscopically. $\mathrm{Ni}$ et al., [13] proposed the key technology of acoustic emission in the study of damage mechanism of composite materials, and pointed out the future development trend. However, there is little research on the acoustic emission characteristics of civil GFRP material.

In this paper, the whole process of GFRP tube under static loading is detected by using acoustic emission instrument. The acoustic source signal collected is processed by using the principle of acoustic emission damage location. The failure process of GFRP tube is reproduced, and the three-dimensional real-time display of damage location is realized.

\section{Detection Principle \\ Principle of acoustic emission testing technology}

Acoustic emission (AE) can be summarized as the phenomenon that the stress is generated inside the object due to the change of the object itself or the external force on the object. When the stress exceeds the yield limit of the object material, the object will produce cracks, deformation or fracture, thus instantly releasing the strain energy to produce stress wave. Therefore, AE is also called stress wave emission.

The principle of acoustic emission technology is shown in Fig-1. The stress wave generated by the object after receiving the external environment excitation propagates to the object surface through the internal medium. The acoustic emission sensor detects the mechanical vibration of the object surface, and converts the mechanical vibration into electrical signal 
Yanhua She \& Gaojie Cai, Saudi J Eng Technol, Feb, 2021; 6(2): 32-36

according to the principle of piezoelectric effect. Due to the attenuation of stress wave in the process of propagation, the electrical signal needs to be amplified by the signal amplifier and then transmitted to the data acquisition instrument, and finally transmitted to the computer through the signal line for data processing.

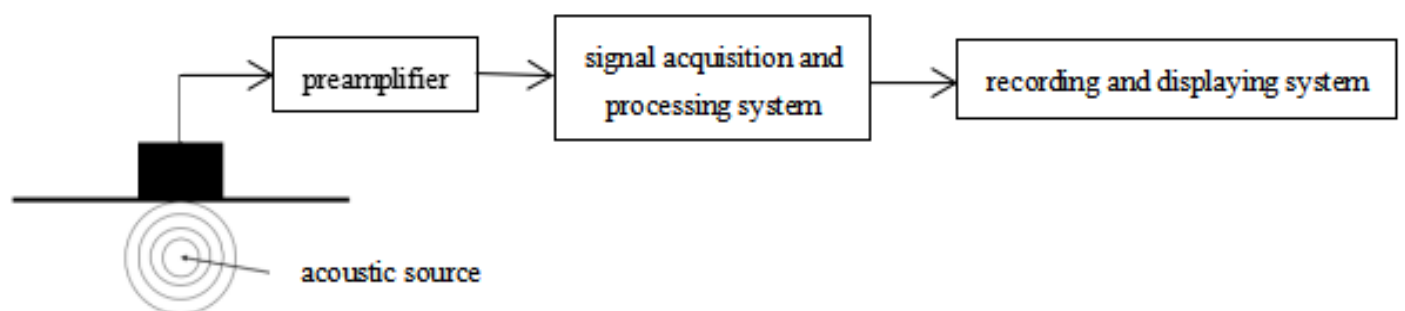

Fig-1: Principle of acoustic emission testing technology

\section{Acoustic emission damage location principle}

In order to locate the coordinates $(\mathrm{x}, \mathrm{y}, \mathrm{z})$ of $\mathrm{AE}$ source in three-dimensional space, it is necessary to know the time ti when the stress wave reaches each sensor and the propagation velocity $\mathrm{v}$ of the stress wave. There are five unknown parameters in total, so at least five sensors are needed to be arranged on the surface of the specimen for three-dimensional positioning. But in the actual test, the velocity of stress wave can be measured by the lead breaking method and acoustic emission instrument, so only four sensors are needed to realize the three-dimensional positioning.

Assuming that the specimen is isotropic material and the wave velocity is uniform, the threedimensional spatial positioning equation is established as follows.

$$
\sqrt{\left(x_{i}-x\right)^{2}+\left(y_{i}-y\right)^{2}+\left(z_{i}-z\right)^{2}}=\left(t_{i}-t\right) v
$$

Where $x_{i}, y_{i}$ and $z_{i}$ are the coordinates of the sensors and $t$ is the arrival time of the stress wave.

\section{Test Overview \\ Specimen Design}

In this test, as shown in Fig-2, the test specimen is the GFRP round pipe with the height of $155 \mathrm{~mm}$, the diameter of $100 \mathrm{~mm}$ and the wall thickness of $5 \mathrm{~mm}$. Because the surface of the specimen is arcshaped, it is not conducive to place the acoustic emission sensor directly. Therefore, a special holder is needed to fix the sensor, as shown in Fig.3. Using epoxy resin as adhesive, the piezoelectric ceramic sensors are arranged on the surface of the specimen, as shown in Fig-4. The location of each sensor is shown in Table-1.

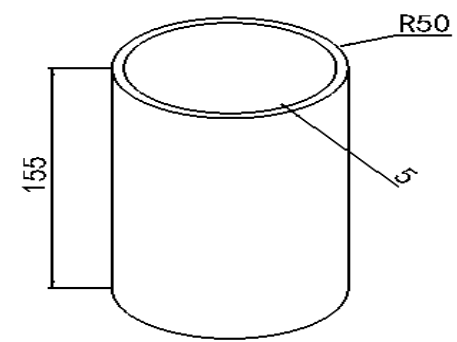

Fig-2: Schematic diagram of GFRP tube specimen (mm)

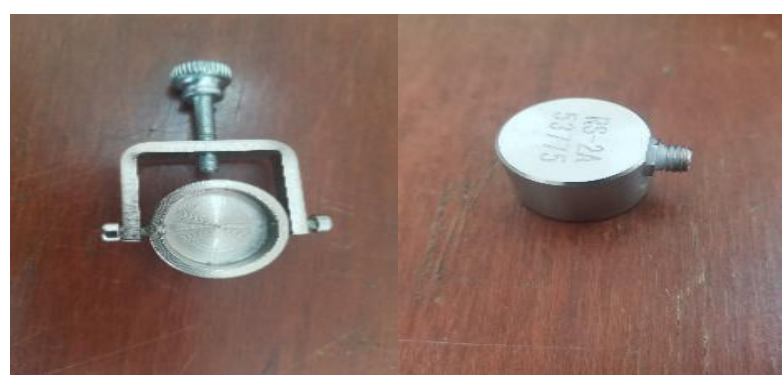

Fig-3: Physical drawing of holder and sensor

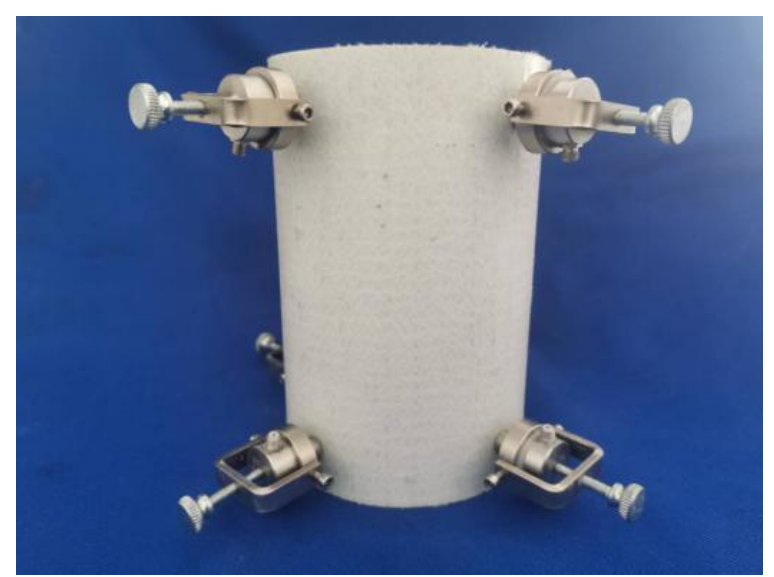

Fig-4: Physical drawing of specimen with sensors

Table-1: Parameters of sensor positions

\begin{tabular}{|l|l|l|l|}
\hline $\begin{array}{l}\text { sensor } \\
\text { number }\end{array}$ & Height(mm) & angle & Radius(mm) \\
\hline 1 & 20 & 0 & 50 \\
\hline 2 & 20 & 90 & 50 \\
\hline 3 & 20 & 180 & 50 \\
\hline 4 & 20 & 270 & 50 \\
\hline 5 & 135 & 0 & 50 \\
\hline 6 & 135 & 90 & 50 \\
\hline 7 & 135 & 180 & 50 \\
\hline 8 & 135 & 270 & 50 \\
\hline
\end{tabular}

\section{Test equipment and process}

The model of acoustic emission data acquisition instrument for test is ds $5-8 b$, as shown in Fig-5. This test needs to damage the specimen by static loading. The static loading device is $300 \mathrm{KN}$ electronic universal testing machine, as shown in Fig-6. 
Before the formal test, the wave velocity is measured on the surface of the specimen by the lead breaking method, and the velocity of the longitudinal wave is $2985 \mathrm{~m} / \mathrm{s}$. Because the specimen is small and the shear wave would not affect the test, the velocity of the transverse wave is set to $2985 \mathrm{~m} / \mathrm{s}$. Then the specimen is put under the static loading device. While loading, acoustic emission instrument is used to collect data, until the specimen is completely destroyed.

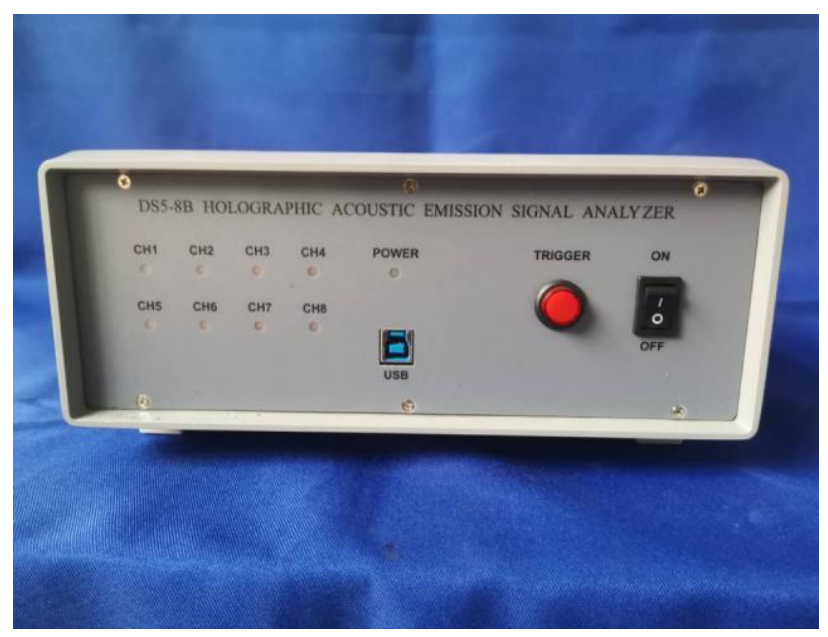

Fig-5: Acoustic emission data acquisition instrument

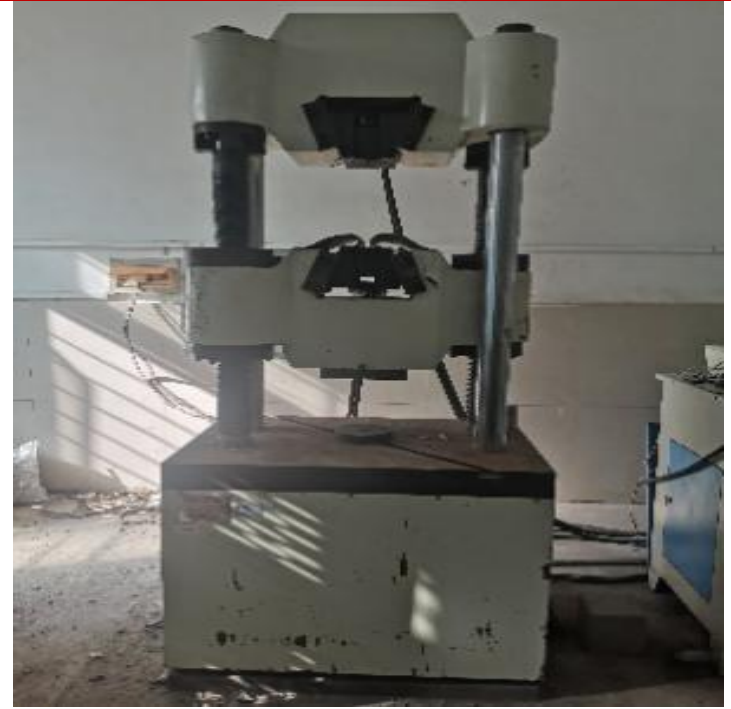

Fig-6: Static loading device

\section{Analysis of Test Results}

During the static loading process of the specimen, obvious cracking sound and cracks appear. As shown in Fig-7, a large area of bulge and cracks appear on the surface of the specimen, and the main locations of cracks are near the sensor 7 .

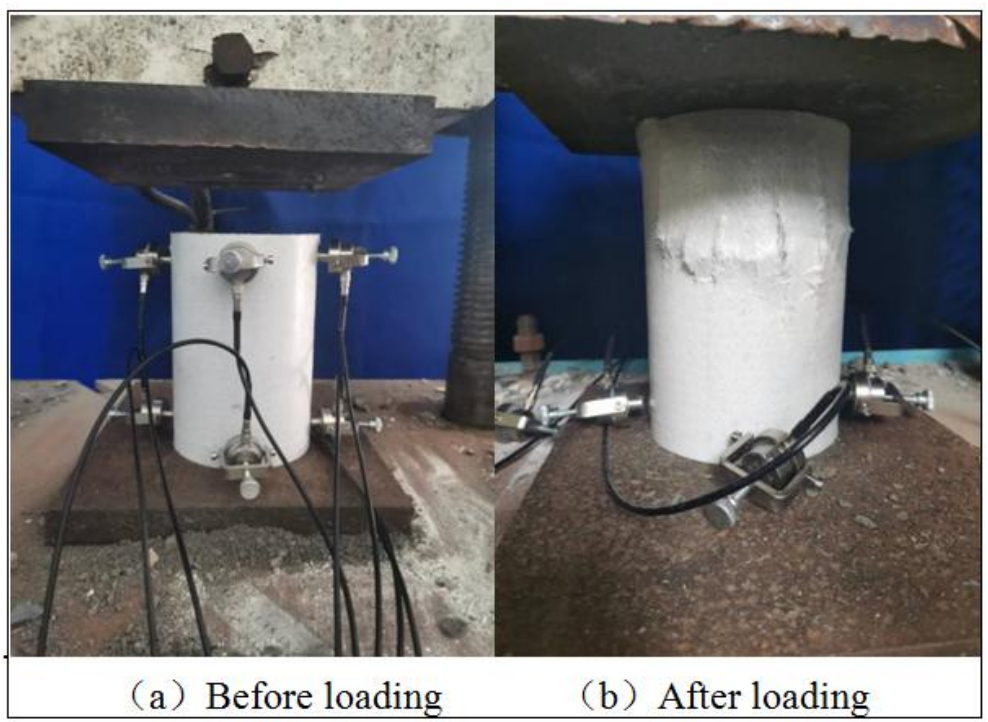

Fig-7: Static loading change of specimen

The load-displacement curve is as shown in Fig-8. With the load increasing, when the load reaches $230 \mathrm{KN}$, the specimen is completely destroyed. The blue dot in the schematic diagram of GFRP tube in the figure is the acoustic emission sensor, and the red dot is the location where the sound source is generated. It can be clearly found that with the increase of load, there are more red points in the figure, and they are mainly concentrated near the sensor 7 . 


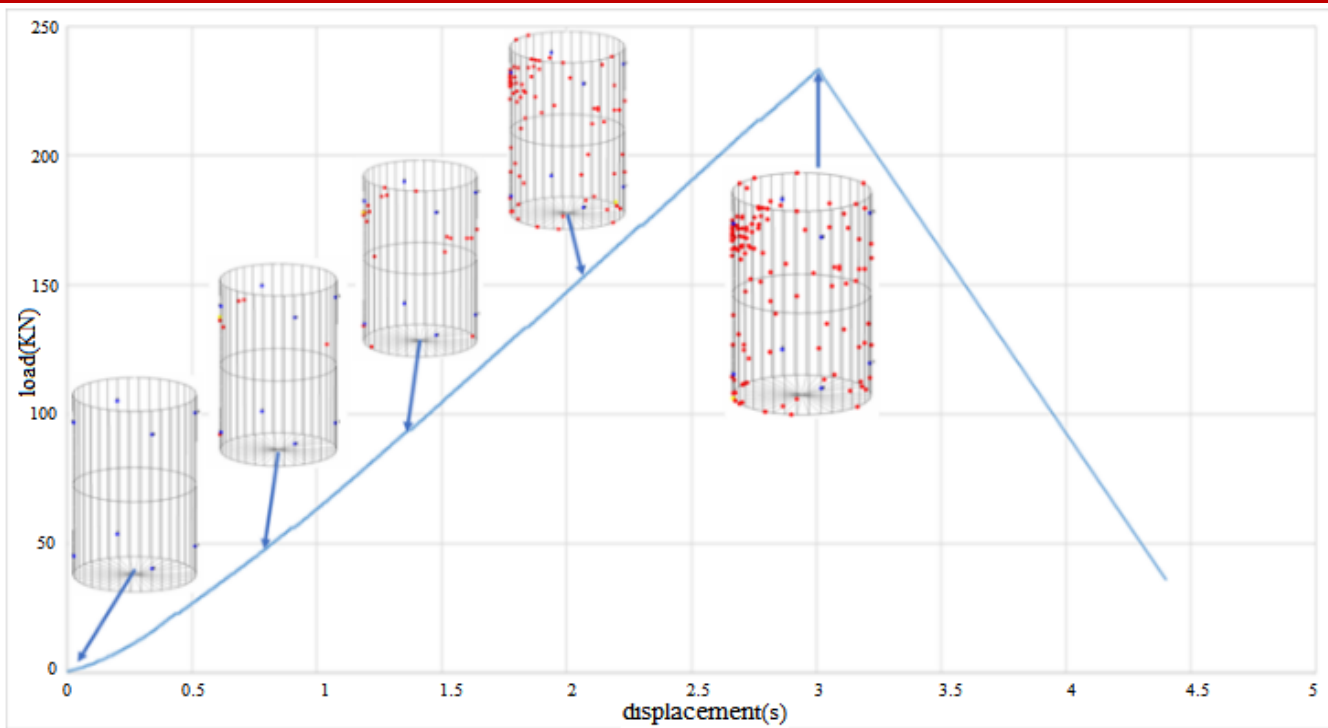

Fig-8: Load-displacement curve

As shown in Fig-9, the white part represents the GFRP tube, the blue point is the location of the sensor, and the red point is the location of the sound source. It can be clearly found that with the gradual increase of load, there are more and more red spots near the sensor 7, which indicates that the sound sources near the sensor 7 become more and more with the increase of load. From Fig-9 (h), it is found that most of the cracks are near the sensor 7 .
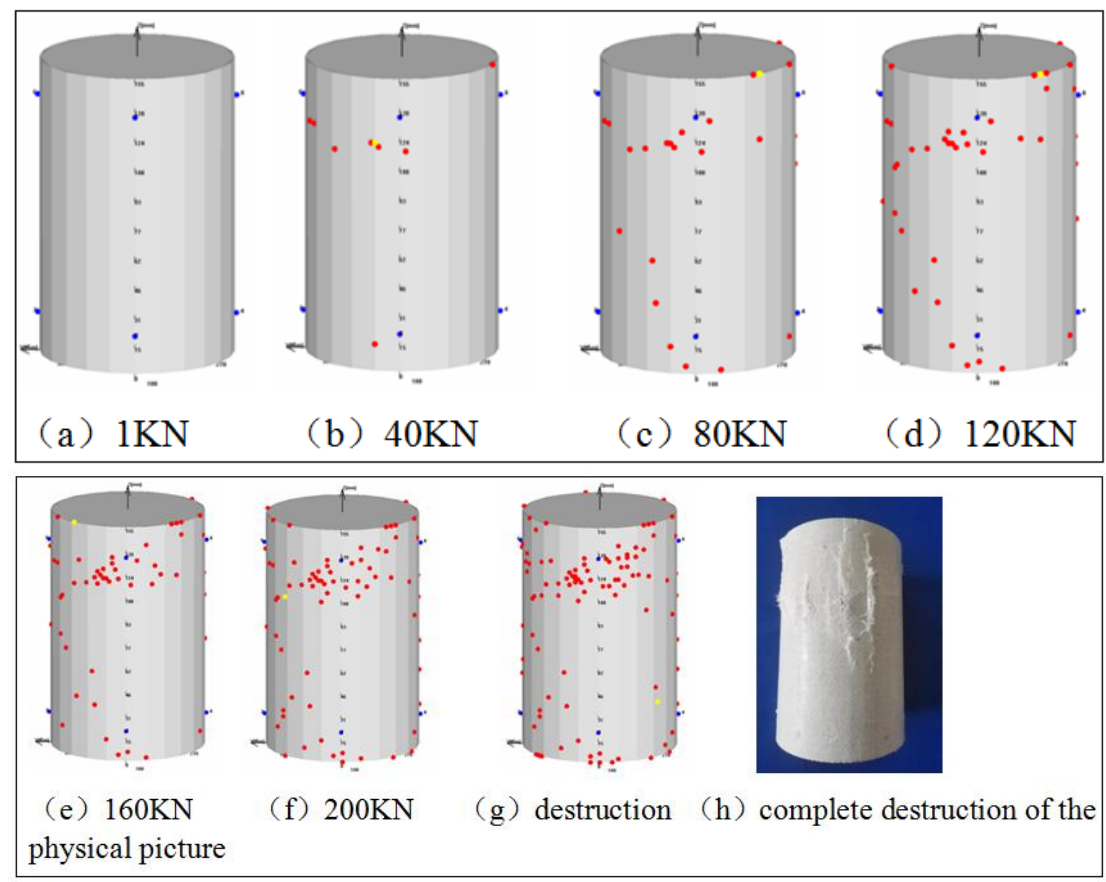

Fig-9: Schematic diagram of sound source location of the sensor 7

As shown in Fig-10, the energy refers to the area under the envelope of acoustic emission waveform signal, which is the reflection of the relative strength of acoustic emission signal. Acoustic emission energy is usually described by mathematical method rather than the real physical energy of acoustic emission signal. Cumulative energy refers to the total energy of acoustic emission signals received in the whole test. The cumulative energy of the sensor 7 is $135715.2 \mathrm{mV}^{*} \mathrm{~ms}$, which is larger than that of other sensors, indicating that the number of sound sources near the sensor 7 is the most, which further reflects that the number of cracks near the sensor 7 is the most. 


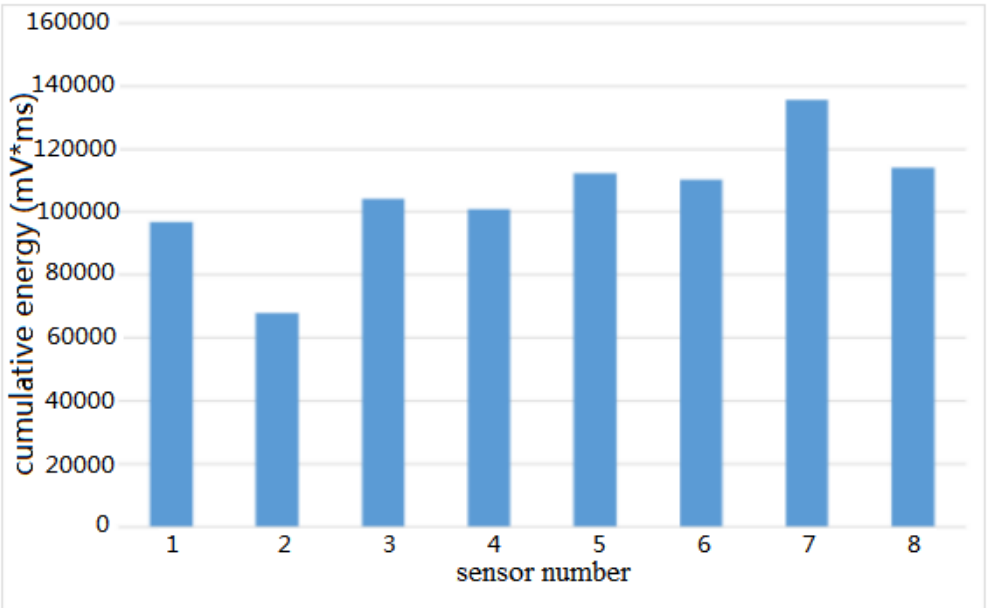

Fig-10: Cumulative energy of acoustic emission signal

In summary, the sound source generated by GFRP pipe under static loading is received by acoustic emission sensor, and data processing is carried out by using acoustic emission damage location principle. Finally, it is concluded that the cracks near the sensor 7 are the most, while in practice, the cracks on the surface of GFRP pipe near the sensor 7 are the most, and the damage is the most serious.

\section{CONCLUSION}

In this paper, acoustic emission technology is used to detect the damage location of GFRP pipe under static loading. The damage location detected by acoustic emission is roughly the same as the actual damage location. The experimental results show that the damage location of GFRP pipe under static loading can be displayed in real time by acoustic emission technology. This not only avoids the complex mathematical calculation, but also improves the efficiency of damage location. The results of location are visualized by three-dimensional images.

\section{ACKNOWLEDGEMENTS}

This work is financially supported by National Natural Science Foundation of China (NSFC, $51408057) \&$ Youth Talent Project of Yangtze University (2015cqr06).

\section{REFERENCES}

1. Wang, L., Jiang, K., \& Liu, Q. C. (2020). Application of GFRP in building energy saving. Technology and Innovation, (13), 99-103.

2. Li, J. W., Qiao, J. G., \& Fu, X. (2019). Research status and Prospect of GFRP anchor in anti floating Engineering. FRP / Composites, (1), 99105.

3. Chen, S., Cai, H. Q., \& Qi, M. (2018). Research status and development of GFRP tube solid concrete composite columns. Jiangxi Building Materials, (4), 24-25.
4. Xu, C. (2015). Technology status and development trend of GFRP glass fiber reinforced polymer bars in Jiangxi building materials. Science and Technology Information, 13(11), 67.

5. Guan, H. (2016). Performance characteristics and development status of FRP pipe. Technology and Enterprise, (4), 218.

6. Xuan, G. Y., Lu, C. H., \& Xu, K. (2020). Experimental study on degradation of tensile properties of GFRP Bars in different erosion environments. Journal of Harbin Institute of Technology, 52(8), 161-168.

7. Yang, W. R., Yuan, J., \& Liang, J. F. (2020). Study on compression membrane effect of steam cured GFRP reinforced concrete slab. Concrete, (11), 112-116.

8. Hadi Muhammad, N. S., Ahmad, H., \& Sheikh, M. N. (2021). Effect of Using GFRP Reinforcement on the Behavior of Hollow-Core Circular Concrete Columns. 25(1).

9. Sangeetha, P., \& Shanmugapriya, M. (2020). GFRP wrapped concrete column compressive strength prediction through neural network. 2(12).

10. Ji, H. G., Wang, J. C., \& Shan, X. Y. (2001). Fractal characteristics of acoustic emission process of concrete and its application in fracture analysis. Journal of Rock Mechanics and Engineering, (6), 801-804.

11. Xu, T., Tang, C. A., \& Zhang, Y. L. (2011). Parallel numerical simulation of debonding failure process of FRP concrete interface. Journal of Solid Mechanics, 32(1), 88-94.

12. Li, W., Wang, Y., \& Wu, C. Q. (2011). Experimental study on damage of FRP composites based on acoustic emission detection technology. Chemical Machinery, 38(6), 692-696.

13. Ni, Y. G., Yang, N., \& Lv, Y. (2019). Application status and development trend of acoustic emission in damage mechanism of composite materials. FRP/Composites, (8), 115-126. 